\title{
Network Reconfiguration in Distribution Systems Using Harmony Search Algorithm
}

\author{
K. Premdeep Reddy ${ }^{1}$, Sabarinath.G ${ }^{2}$, Dr. T. Gowri Manohar ${ }^{3}$ \\ ${ }^{I}(P G$ Student, E.E.E Department, S.V.University, Tirupati, India) \\ ${ }^{2}$ (Research Scholar, E.E.E Department, S.V.University, Tirupati, India) \\ ${ }^{3}$ (Professor, E.E.E Department, S.V.University, Tirupati, India)
}

\begin{abstract}
This manuscript explores feeder reconfiguration in distribution networks and presents an efficient method to optimize the radial distribution system by means of simultaneous reconfiguration. Network Reconfiguration of radial distribution system is a significant way of altering the power flow through the lines. This assessment presents a modern method to solve the network reconfiguration problem with an objective of minimizing real power loss and improving the voltage profile in radial distribution system (RDS). A precise and load flow algorithm is applied and the objective function is formulated to solve the problem which includes power loss minimization. HSA Algorithm is utilized to restructure and identify the optimal strap switches for minimization of real power loss in a distribution network. The strategy has been tested on IEEE 33-bus and 69bus systems to show the accomplishment and the adequacy of the proposed technique. The results demonstrate that a significant reduction in real power losses and improvement of voltage profiles.
\end{abstract}

Keywords: Harmony Search Algorithm, load flow analysis, Network Reconfiguration, real power loss, Radial distribution systems, voltage profile.

\section{Introduction}

A power distribution network consists of a group of radial feeders which can be connected together with several tie switches and tie lines. Power loss reduction in the network is a major concern of electric distribution utilities. Among conventional methods, optimal reconfiguration and capacitor placement are two effective methods which can be applied to the network. Optimization methods have been used to find the optimal location and size of different devices such as capacitors, FACTS, and distributed generations, in power distribution systems. In this paper, optimization methods are used for optimal reconfiguration and capacitor allocation in power systems. Feeder reconfiguration is the process of changing the distribution network topology by changing the status of its switches. There are two kinds of switches based on their open or close conditions: normally open (N.O.) and normally close (N.C.). N.O. Switches are considered as tie switches in the network. During a reconfiguration process, the status of these switches will be changed optimally based on the proposed objective function. Opening and closing of these switches can change the amount of power losses.

Optimal capacitor placement and distribution network feeder reconfiguration (DNR) have been investigated in many papers separately, and various approaches have been used with different objective functions to model the network and optimize the problem solution. A number of research papers have been emerged on the general topic of feeder reconfiguration; there is still a need to develop more appropriate and effective techniques for the reconfiguration under reliable operating conditions.Many recent researches on distribution feeder reconfiguration on reducing the power losses. Baran and $\mathrm{Wu}$ [1], described the optimal size of capacitors, so that the real power losses will be minimized for a given load profile. This method is efficient, numerically robust and has good convergence characteristics. Sundhararajan [2], proposed to minimize the energy losses by sensitivity analysis to select locations and genetic algorithm is used to determine the optimal capacitor locations. Das [3], presented genetic algorithm is to select the optimal values of switched capacitors required to place on the radial system under varying load conditions to minimize the energy loss while keeping the voltage at load buses within prescribed limits. Milosevic [4], proposed a strategy for placing capacitors at multiple locations on a distribution feeder to power factor correction and power loss reduction. Damodar Reddy [5], a fuzzy multi-objective algorithm is used to solve an optimal capacitor placement and reconfiguration. Abdellatif [6], a simple and efficient load flow solution has been proposed for determining voltage rms values and phase-angles of radial distribution feeders. Hooshmand [7], multi-objective problem, bacterial foraging oriented by PSO algorithm, a new method for the optimal location of fixed and switching capacitors were presented to reduce the costs of power losses as well as improving the voltage profile. Damodar Reddy [8-9] a two-stage methodology of finding the optimal locations and sizes of shunt capacitors for loss reduction in radial distribution systems is presented. Fuzzy approach is proposed to find the optimal capacitor locations and Harmony Search Algorithm is proposed to find the optimal capacitor sizes. Farahani [10], Reconfiguration is based on a simple branch exchange method of a single loop. A joint optimization algorithm is proposed for 
combining this improved method of reconfiguration and capacitor placement for maximum loss reduction. ElFergany [11], an efficient heuristic based approach to assign static shunt capacitors using an ABC algorithm to enhance overall system static voltage stability index and achieve maximum savings. Sravan Reddy [12], proposed a hybrid method to determine the optimal capacitor placement in distribution networks by using fuzzy and shuffled frog leaping algorithm. Sudhakar Reddy [13] proposed dragonfly algorithm to resolve the final network reconfiguration and reduce the system losses. Sudhakar Reddy [14-15] proposed PSO algorithm to determine the optimal network reconfiguration to reduce the real power losses and variational technique is used to sizing for D-STATCOM. Bhargav Reddy [16] proposed PSO algorithm to determine the different configurations to reduce the real power losses at different load conditions.

\subsection{Calculation of load current:}

\section{Problem Formulation}

The complex power injected into the bus ${ }^{n}$ is given by

$\mathrm{S}_{\mathrm{n}}=\mathrm{P}_{\mathrm{n}}+\mathrm{jQ} \mathrm{Q}_{\mathrm{n}}=\mathrm{V}_{\mathrm{n}} \mathrm{I}_{\mathrm{n}}^{*}$

The load current at any bus $\mathrm{n}_{\text {is given by }} \mathrm{I}_{\mathrm{L}, \mathrm{n}}=\left(\frac{\mathrm{P}_{\mathrm{n}}+\mathrm{jQ} \mathrm{n}}{\mathrm{v}_{\mathrm{n}}}\right)^{*}=\frac{\mathrm{P}_{\mathrm{n}}-\mathrm{jQ} \mathrm{n}}{\mathrm{v}_{\mathrm{n}}^{*}}$

Where $\mathrm{n}=1,2,3, \ldots \ldots \mathrm{N}$

Where, $\mathrm{N}=$ total number of buses
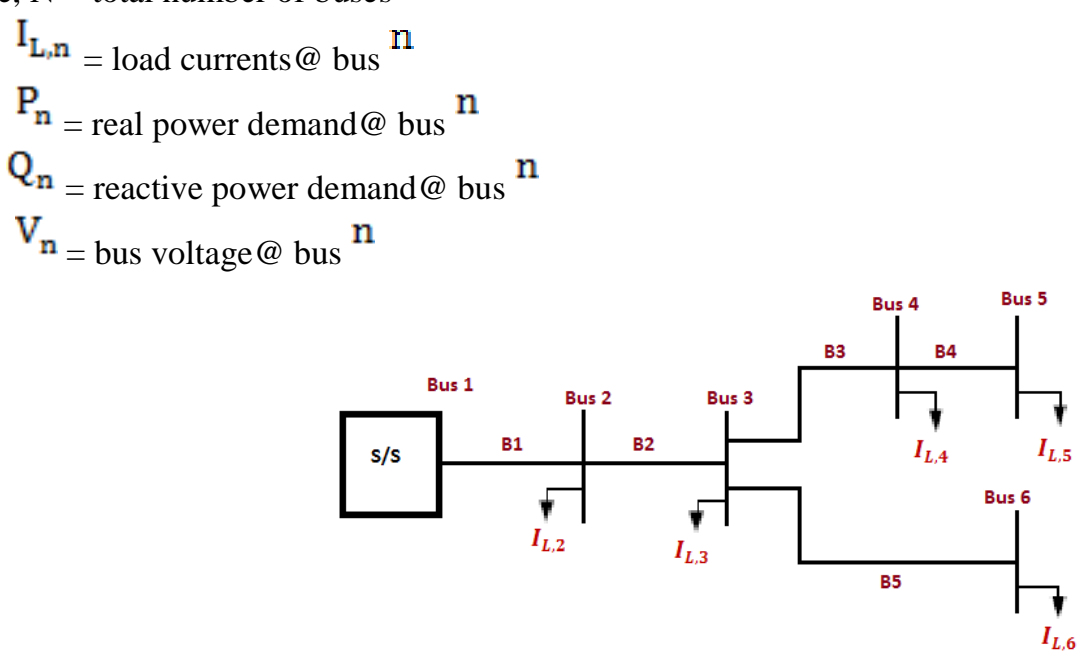

Fig 1: A Simple 6-bus radial distribution system

\subsection{Formation of BIBC matrix:}

The relation between load currents and branch currents can be found by using KCL equations as follows.

$$
\begin{aligned}
& I_{B 1}=I_{L 2}+I_{L 3}+I_{L 4}+I_{L 5}+I_{L 6} \\
& I_{B 2}=I_{L 3}+I_{L 4}+I_{L 5}+I_{L 6} \\
& I_{B 3}=I_{L 4}+I_{L 5} \\
& I_{B 4}=I_{L 5} \\
& I_{B 5}=I_{L 6}
\end{aligned}
$$

Thus the relationship between load currents and branch currents can be expressed in matrix form as shown below.

$$
\left[\begin{array}{l}
\mathrm{I}_{\mathrm{B} 1} \\
\mathrm{I}_{\mathrm{B} 2} \\
\mathrm{I}_{\mathrm{B} 3} \\
\mathrm{I}_{\mathrm{B} 4} \\
\mathrm{I}_{\mathrm{B} 5}
\end{array}\right]=\left[\begin{array}{lllll}
1 & 1 & 1 & 1 & 1 \\
0 & 1 & 1 & 1 & 1 \\
0 & 0 & 1 & 1 & 0 \\
0 & 0 & 0 & 1 & 0 \\
0 & 0 & 0 & 0 & 1
\end{array}\right]\left[\begin{array}{l}
\mathrm{I}_{\mathrm{L} 2} \\
\mathrm{I}_{\mathrm{L} 3} \\
\mathrm{I}_{\mathrm{L} 4} \\
\mathrm{I}_{\mathrm{L} 5} \\
\mathrm{I}_{\mathrm{L} 6}
\end{array}\right]
$$

The above matrix is reduced to 
$[\mathrm{IB}]=[\mathrm{BIBC}][\mathrm{IL}]$

The receiving end voltages can be premeditated by forward sweeping across the line by subtracting the line section drop from the sending end voltages of the line section.

$V_{\mathrm{q}}(\mathrm{K})=\mathrm{V}_{\mathrm{p}}(\mathrm{K})-\mathrm{I}_{\mathrm{B}}(\mathrm{K})_{*} \mathrm{Z}_{\mathrm{p}}(\mathrm{K})$

The power losses in the distribution systems are

$P_{L T}=\sum_{i=1}^{b} I_{i}^{2} R_{i}$

\subsection{Objective Function}

The objective function of the problem is formulated to exploit the power loss reduction in the radial distributed system, which is given by

Fitness function $=\min \left\{P_{\text {loss }}\right\}$

\section{Harmony Search Algorithm}

The harmony search algorithm (HSA) is a new meta-heuristic algorithm. The harmony search algorithm (HSA) is simple in concept, few in parameters and easy in implementation. The Harmony search algorithm is the concept of natural musical performance processes. The musicians starting with some harmonies, they attempt to achieve better harmonies by improvisation and create iteratively new best solutions based on past solutions on random modifications. Finally HSA gives optimum value. This algorithm was originally developed for discrete optimization and later expanded for continuous optimization. It has been successfully applied to various computational optimization problems such as structural design, water network design, dam scheduling, school bus routing, Sudoku game, musical composition, benchmark and real-world problems.

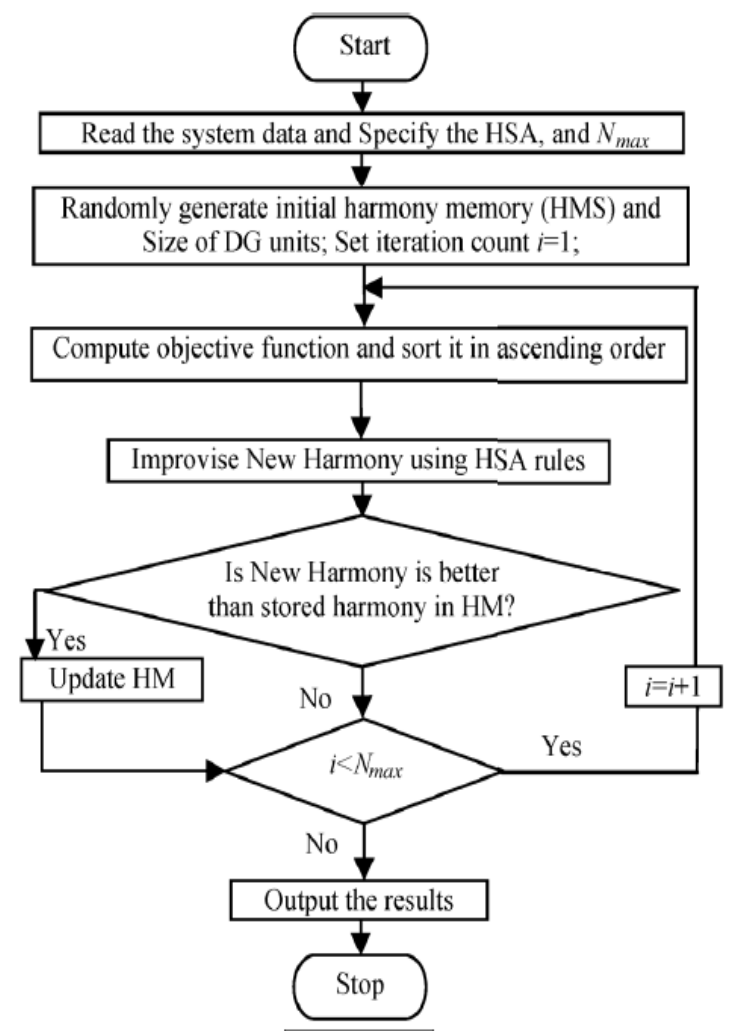

Fig 2: Flow chart for Proposed Algorithm

The HS algorithm initializes the Harmony Memory (HM) with randomly generated solutions. The number of solutions stored in the HM is defined by the Harmony Memory Size (HMS). Then iteratively a new solution is created as follows. Each decision variable is generated either on memory consideration and a possible additional modification, or on random selection. The parameters that are used in the generation process of a new solution are called Harmony Memory Considering Rate (HMCR) and Pitch Adjust Rate (PAR). After a new solution has been created, it is evaluated and compared to the worst solution in the HM. If its objective value is better than that of the worst solution, it replaces the worst solution in the HM. This process is repeated, until a termination criterion is fulfilled. 
Application of HSA for loss minimization problem with reconfiguration is illustrated with the help of standard 33-bus radial distribution system. In 33-bus system, there are five open tie switches with branch numbers 33, 34, 35, 36, and 37, respectively, which forms five loops to as shown in Fig. 3. Further, assume that candidate buses for optimal installation of DG units, which is not proposed in this work.

\subsection{Algorithm to find the optimum reconfiguration using harmony search algorithm}

Step 1) Initialize the problem and algorithm parameters.

Step 2) Initialize the harmony memory.

Step 3) Improvise a new harmony.

Step 4) Update the harmony memory.

Step 5) Check the termination criterion.

The solution vector corresponding to the best fitness value gives the optimal network RCG in n optimal switching. Apply Greedy Search between old harmony and New Harmony by comparing fitness values. The convergence pattern is presented and the method was tested on several other systems and yields a good performance. It may also be noted that the HSA algorithm takes a small solution time. This may be achieved through appropriate tuning. Appropriate tuning may be done through proper selection of Harmony Memory Considering Rate (HMCR) and Pitch Adjust Rate (PAR).

In the present work, harmony memory size $\mathrm{HMS}=50, \mathrm{HMCR}=0.9$; harmony consideration rate $0<\mathrm{HMCR}<1$; PARmin=0.4; PARmax=0.9; min bandwidth, bwmin=0.0001; $\max$ bandwidth bwmax=1.0; maximum no of iterations $=1000$.

\section{Results}

In order to demonstrate the effectiveness of the anticipated method, HSA is tested on standard IEEE 33-bus and 69-bus systems.

\section{3-bus Test System:}

The 33-bus system is a large-scale radial distribution system with 32 sectionalizing and five tie switches. Configuration, line, load, and tie line data are taken from [14]. Total system loads for base configuration are $3715 \mathrm{~kW}$ and $2300 \mathrm{kVAR}$. The sectionalizing switches are labeled from 1 to 32 and tie switches from 33 to 37 , respectively. HSA parameters of the algorithm used to simulate this test system and results are shown for test system in table 1 .

Table 1: Network Reconfiguration Results for 33 bus systems

\begin{tabular}{|l|l|l|}
\hline 1 & Initial configuration & $33,34,35,36,37$ \\
\hline 2 & Total real power loss of the original network & $\mathbf{1 8 2 . 1 7 6 0} \mathbf{~ W W}$ \\
\hline 3 & Total reactive power loss of the original network & $123.2556 \mathrm{kVAR}$ \\
\hline 4 & Minimum voltage of the original network & $0.9115 \mathrm{p.u}$ \\
\hline 5 & Final reconfiguration & $11,14,7,31,28$ \\
\hline 6 & Real power loss for the final reconfigured network & $\mathbf{8 7 . 5 6 2 3} \mathbf{~ k W}$ \\
\hline 7 & Total reactive power loss of the final reconfigured network & $64.6136 \mathrm{kVAR}$ \\
\hline 8 & Minimum voltage of the final reconfigured network & $0.9557 \mathrm{p.u}$ \\
\hline 9 & Loss reduction for final reconfiguration & $\mathbf{5 1 . 9 3 \%}$ \\
\hline
\end{tabular}

\section{9-bus Test System:}

The 69-bus system is a large-scale radial distribution system with 68 sectionalizing and five tie switches. Configuration, line, load, and tie line data are taken from [14]. Total system loads for base configuration are $3802.2 \mathrm{~kW}$ and $2694.6 \mathrm{kVAR}$. The sectionalizing switches are labeled from1 to 68 and tie switches from 69 to 73, respectively. HSA parameters of the algorithm used to simulate this 69-bus test system and results are shown for test system in table 2.

Table 2: Network Reconfiguration Results for 69 bus systems

\begin{tabular}{|c|c|c|}
\hline 1 & Initial configuration & $\begin{array}{lllll}69 & 70 & 71 & 72 & 73\end{array}$ \\
\hline 2 & Total real power loss of the original network & $224.9767 \mathrm{~kW}$ \\
\hline 3 & Total reactive power loss of the original network & $102.1548 \mathrm{kVAR}$ \\
\hline 4 & Minimum voltage of the original network & 0.9092 p.u \\
\hline 5 & Final reconfiguration & $\begin{array}{lllll}69 & 70 & 14 & 58 & 61 \\
\end{array}$ \\
\hline 6 & Real power loss for the final reconfigured network & $99.6195 \mathrm{~kW}$ \\
\hline 7 & Total reactive power loss of the final reconfigured network & $114.6828 \mathrm{kVAR}$ \\
\hline 8 & Minimum voltage of the final reconfigured network & 0.9428 \\
\hline 9 & Loss reduction for final reconfiguration & $55.72 \%$ \\
\hline
\end{tabular}




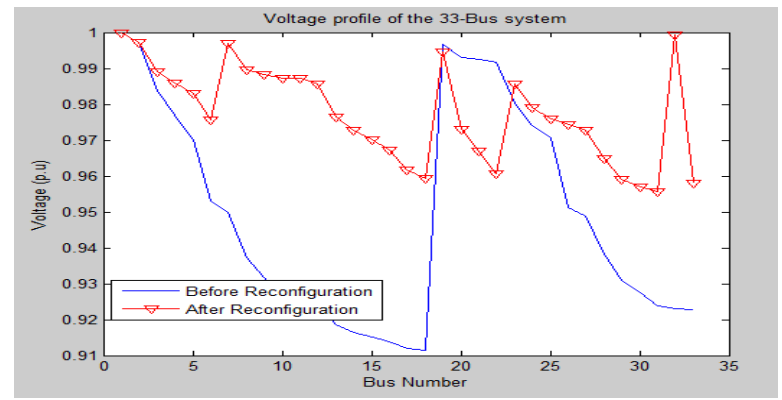

Fig. 3: Comparison of voltages before and after final reconfiguration for 33-bus system

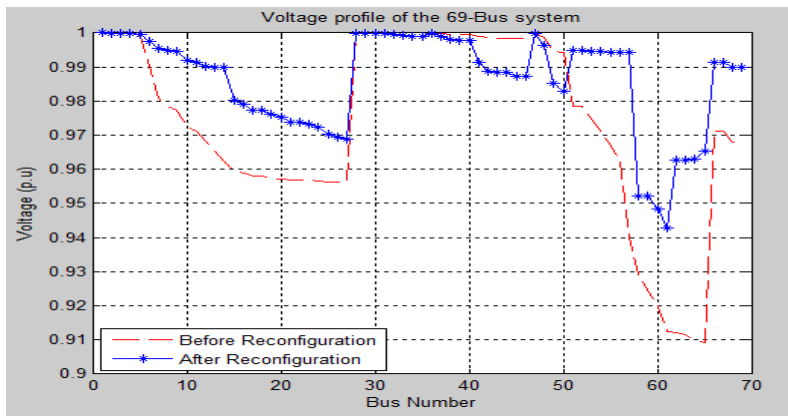

Fig. 4: Comparison of voltages before and after final reconfiguration for 69-bus system

\section{Conclusion}

In this paper, the HSA algorithm is successively applied to a distribution feeder problem. The objective to minimize the real power loss, while maintaining the radial structure of the network based on, LSW33 and LSW69 [12]. The effectiveness of the proposed method is demonstrated on 33-bus and 69-bus systems. The numerical results illustrate that the proposed algorithm is capable of finding the optimal solution is obtained by using an HSA. Thus, the final results of the proposed HSA method illustrate considerable loss reduction is possible as compared to previous reports of other researchers. The active power loss reduction for IEEE 33-bus and 69-bus systems are $51.93 \%$ and $55.72 \%$ respectively.

\section{References}

[1] M. E. Baran and F. Wu, "Optimal sizing of capacitors placed on a radial distribution system" IEEE Trans. Power Del., vol. 4, no. 1, pp.735-743, Jan. 1989.

[2] Srinivasan Sundhararajan, Ani1 Pahwa, "Optimal selection of capacitors for radial distribution systems using a genetic algorithm", IEEE Transactions on Power Systems, Vol.9, No.3, pp.1499-1507, August 1994.

[3] D.Das, "Reactive power compensation for radial distribution networks using genetic algorithm", Electrical power and Energy systems, 24, pp.573-581, 2002.

[4] Borka Milosevic, Miroslav Begovic, "Capacitor placement for conservative voltage reduction on distribution feeder", IEEE Transactions on Power Delivery, Vol.19, No.3, pp.1360-1367, July 2004.

[5] M. Damodar Reddy, Prof. V.C.Veera Reddy "A two-stage methodology of optimal capacitor placement for the reconfigured network", Indian Journal of Engineering \& Material Sciences, Vol-17, p.p. 105-112, April - 2010.

[6] Abdellatif Hamouda, Khaled Zehar, "Improved algorithm for radial distribution network load flow solution", Electrical Power and Energy Systems 33, pp.508-514, 2011.

[7] Rahamat Allah Hooshmand, Hossein Mohkam, "New optimal placement of capacitors and dispersed generators using bacterial foraging oriented by particle swarm optimization algorithm in distribution systems", Springer, 2011.

[8] M. Damodar Reddy and Dr. V.C. Veera Reddy, "A two-stage methodology of network reconfiguration for the compensated network", i-manager's Journal on Electrical Engineering, Vol-1, No-4, p.p. 22-29, April-June 2008

[9] M. Damodar Reddy, N.V.Vijay Kumar "Optimal Capacitor Placement for Loss Reduction in Distribution System Using Fuzzy and Harmony Search Algorithm”, ARPN Journal of Engineering and Applied Sciences, Vol. 7, No.1, pp. 15-19, January - 2012.

[10] Vahid Farahani, Behrooz Vahidi, Hossein Askarian Abyaneh, "Reconfiguration and Capacitor Placement Simultaneously for Energy Loss Reduction Based on an Improved Reconfiguration Method", IEEE Trans. on Power Systems, Vol.27, iss.2, 2012.

[11] Attia El-Fergany, A.Y.Abdelaziz "Improved for radial distribution networks using Artificial Bee Colony Algorithm", Journal of Electrical Engg Technology Vol 9, No. 2, pp.441-451, 2014.

[12] K. Sravan Kumar Reddy, Prof. M. Damodar Reddy, "Optimal Placement of Capacitor in Distribution Networks using Fuzzy and SFLA" IEEE International Conference on Electrical, Electronics, Signals, Communication and Optimization (EESCO) - 2015.

[13] A. V. Sudhakara Reddy, Prof. M. Damodar Reddy, "Optimization of distribution network reconfiguration using a dragonfly algorithm”, Journal of Electrical Engineering,Dec- 2016.

[14] A. V. Sudhakara Reddy, Prof. M. Damodar Reddy, "Optimization of network reconfiguration by using particle swarm optimization”, IEEE, Delhi Technological University, 2016.

[15] A. V. Sudhakara Reddy, Prof. M. Damodar Reddy, "Optimal network reconfiguration and D-STATCOM allocation in primary distribution system ",DIET, Visakhapatnam, Andhra Pradesh, India, ICAMT-2016.

[16] P.Prasad, B.Bhargav Reddy, "Power loss minimization in distribution system using network reconfiguration with particle swarm optimization" International Journal of Engineering Science \& Advanced Technology, Vol.-5 issue-3, pp 171-178 June 2015. 